\title{
Autoimmuninduktion durch pegyliertes Interferon-alpha-2b in Korrelation zur Überlebenszeit bei Mittel- und Hochrisikomelanompatienten
}

\author{
Induction of Autoimmunity by Pegylated Interferon-alpha-2b in Correlation to Survival Time in Medium \\ and High Risk Melanoma Patients
}

\section{Autoren}

Institute

\section{Loquai' ${ }^{1}$ B. Schlüter ${ }^{2}$, H. W. Klötgen ${ }^{3}$, T. A. Luger ${ }^{4}$, S. Grabbe' ${ }^{1}$, D. Nashan}

Klinik und Poliklinik für Dermatologie, Universität Mainz

Institut für klinische Chemie und Laboratoriumsmedizin, Universität Münster

Klinik und Poliklinik für Dermatologie, Venerologie und Allergologie, Universität Duisburg-Essen

Klinik und Poliklinik für Dermatologie, Allergologie und Venerologie, Universität Münster

Klinik für Dermatologie, Universität Freiburg

\section{Bibliografie}

DOI $10.1055 / \mathrm{s}-2007-995760$

Akt Dermatol 2008; 34 :

170-174 @ Georg Thieme

Verlag KG Stuttgart · New York ISSN 0340-2541

\section{Korrespondenzadresse}

Dr. med. Carmen Loquai

Klinik und Poliklinik für

Dermatologie

Universität Mainz

Langenbeckstr. 1

55131 Mainz

carmen.loquai@ukmainz.de

\section{Zusammenfassung \\ $\nabla$}

Hintergrund: Das Auftreten von Autoimmunreaktionen bei Tumorpatienten scheint mit einer besseren Prognose assoziiert zu sein. Ziel dieser retrospektiven Datenanalyse war die Erfassung von Autoimmunreaktionen unter Therapie mit pegyliertem Interferon-alpha-2b und die Untersuchung einer Korrelation zwischen Autoimmuninduktion und Überlebenszeit.

Patienten und Methodik: Hierzu wurden bei 25 Patienten mit histologisch nachgewiesenem malignem Melanom im R0-resezierten Stadium IbIV vor Therapiebeginn sowie alle drei Monate während einer 18-monatigen adjuvanten Therapie mit $2 \mu \mathrm{g} / \mathrm{kg}$ pegyliertem Interferon-alpha- $2 \mathrm{~b}$ pro Woche klinische und serologische Parameter zur Detektierung einer Autoimmunerkrankung erhoben.

Ergebnisse: Unter Therapie entwickelten 16 von 25 Patienten (64\%) Autoantikörper. Die Überlebenszeiten der Patienten mit nachweislich neuen

\section{Einfithrung}

$\nabla$

Interferon-alpha (IFN- $\alpha)$ ist in Deutschland seit Juni 1997 für die adjuvante Behandlung des malignen Melanoms zugelassen. Die Akzeptanz der adjuvanten IFN-Therapie mit ihrer nicht unerheblichen Toxizität und hohen Kosten wäre zu steigern, wenn Patienten, die von einer solchen Therapie profitieren würden, genau definiert und selektiert werden könnten.

Immunologische Mechanismen, die in der IFNTherapie eine Rolle spielen, umfassen unter anderem eine verstärkte Antigenpräsentation infolge der Hochregulation von Major-Histokompatibilitätskomplex Molekülen (MHC), die Induktion dendritischer Zellen, eine Funktionssteigerung immunkompetenter Effektorzellen, antiangioge-
Autoantikörpern und solche ohne Entwicklung von Autoantikörpern unterschieden sich nur unwesentlich voneinander (31,6 versus 31,9 Monate). Alle 6 Patienten, die unter Interferontherapie eine klinisch manifeste Autoimmunthyreoiditis und/oder spezifische antinukleäre Antikörper entwickelten, waren jedoch 36 Monate nach Beginn der Therapie noch am Leben, davon zwei Patienten in kompletter Remission im Stadium IV. Verglichen hierzu lag die mittlere Überlebenszeit in der AK-positiven physiologisch irrelevanten Restgruppe bei 26,73 Monaten ( $n=10 / 16)$.

Schlussfolgerung: Die Anzahl unserer Patienten, die eine Autoimmunreaktion unter pegyliertem Interferon-alpha-2b entwickelten, ist verglichen mit publizierten Daten zum nicht pegylierten Interferon-alpha deutlich höher. Die Relevanz spezifischer Autoimmunreaktionen als prognostischer Marker einer krankheitskontrollierenden antitumorösen Immunantwort bleibt zu beweisen.

netische Effekte und direkt antiproliferative Effekte auf den Tumor.

Von den zahlreichen IFN-Nebenwirkungen treten Autoimmunphänomene und Autoimmunerkrankungen wie Thyreoiditis oder Vitiligo bei $15 \%$ bis $30 \%$ der Patienten auf [1 -4]. Bei einigen Erkrankungen wie chronischen Virushepatitiden oder myeloproliferativen Erkrankungen konnten schon vor Beginn der IFN-Therapie erhöhte Autoantikörpertiter und Autoimmunerkrankungen nachgewiesen werden [5-7].

Eine Korrelation zwischen antitumoraler Antwort und Autoimmunität ist bekannt [8]. Analog zur antitumoralen Wirkung und als Indiz für die Immunstimulation könnte die Immunantwort gegen Autoantigene auch beim Melanom zu werten sein. Erst kürzlich konnten Gogas et al. nach- 
weisen, dass das Auftreten von Autoantikörpern (Auto-AK) oder die klinische Manifestation einer Autoimmunität während einer adjuvanten Hochdosis-IFN-Therapie des malignen Melanoms mit einer statistisch signifikanten Verbesserung des rezidivfreien und Gesamtüberlebens einhergeht [9]. Auch andere immunmodulierende Substanzen wie Interleukin-2 oder anti-CTLA-4 AK zeigen eine Korrelation zwischen Tumorregression und Therapie-induzierter Autoimmunerkrankung [10,11].

Pegyliertes IFN- $\alpha-2 b$ (PEG-IFN- $\alpha-2 b$ ) (PegIntron; Schering Corporation, Kenilworth, NJ) ist ein Derivat des rekombinanten IFN$\alpha$-2b gekoppelt an ein Einzelstrangpolyethylenglykol mit einem mittleren Molekulargewicht von 12000 Dalton. Die Pegylierung bleibt ohne Einfluss auf die Tertiärstruktur oder das Aktivitätsspektrum, erhöht aber die Plasmahalbwertszeit von IFN- $\alpha$ auf das Zehnfache [12]. Dies hat zur Folge, dass PEG-IFN- $\alpha-2 b$ nur einmal wöchentlich verabreicht werden muss. Im Vergleich zum nicht pegylierten IFN- $\alpha-2 b$ bedeutet eine verminderte Injektionsfrequenz bei erhöhter AUC und somit erhöhter Medikamentenexposition für den Patienten eine deutliche Verbesserung der Lebensqualität.

Die umfangreichsten Erfahrungen mit PEG-IFN- $\alpha$-2b liegen in der Therapie der chronischen Hepatitis $C$ vor. Aufgrund seiner signifikant verbesserten antiviralen Aktivität hat pegyliertes IFN- $\alpha$ in der Hepatitisbehandlung die nicht pegylierten Formen verdrängt $[13,14]$. Phase I/II Studien mit PEG-IFN- $\alpha$-2b bei der chronisch myeloischen Leukämie, aber auch bei soliden Tumoren wie dem Nierenzellkarzinom und beim malignen Melanom, deuten ebenfalls auf ein verbessertes Ansprechen bei vergleichbarem Nebenwirkungsprofil hin, ohne dass gezielte Erklärungen dafür bekannt sind $[15,16]$.

Bisher wurde nicht untersucht, ob und inwiefern die Pegylierung des IFN- $\alpha$ einen Einfluss auf die Induktion einer Autoimmunantwort im Vergleich zu nicht pegyliertem IFN- $\alpha$ besitzt.

In dieser retrospektiven Analyse wurde eine differenzierte Erfassung unter einer PEG-IFN- $\alpha$-Therapie induzierter Autoantikörper zusammengestellt und in Korrelation mit dem Tumorverlauf gestellt wie auch mit Literaturdaten verglichen.

\section{Patienten und Methoden}

$\nabla$

Es erfolgte eine retrospektive Datenanalyse von 25 Patienten mit histologisch nachgewiesenem malignem Melanom im R0-resezierten AJCC Stadium Ib-IV, bei denen in einem Zeitraum von 2 Jahren eine primäre adjuvante Immuntherapie mit PEG-IFN$\alpha-2 b$ begonnen worden war. Patienten im Stadium Ib hatten die Immuntherapie aufgrund eines erhöhten Risikoprofils erhalten (Schleimhautmelanom, auswärts primär nicht in toto kurretiertes Melanom, Rezidivtumor). Patienten im Stadium IV erhielten die adjuvante Immuntherapie im Stadium der kompletten Remission. Die Charakteristika der Patienten sind in $\bullet$ Tab. 1 aufgelistet. Kontraindikationen einer adjuvanten Therapie mit PEGIFN- $\alpha$-2b waren schwerwiegende kardiale Vorerkrankungen, Niereninsuffizienz, Leberinsuffizienz, schwere Lungenerkrankungen oder instabile psychiatrische Erkrankungen. Zusätzlich wurden Patienten mit unbehandelten Infektionen, mit Nachweis von HIV-AK, HBs- oder HBc-Antigen sowie mit vorbestehenden Autoimmunerkrankungen von der Behandlung ausgeschlossen. Patienten mit vorangegangener chemo- oder immuntherapeutischer Therapie gingen in die Datenanalyse nicht ein. Der Abschluss der vorangegangenen operativen Melanomtherapien durfte nicht länger als acht Wochen zurückliegen. Nach Aufklä-

\begin{tabular}{|c|c|c|c|}
\hline & Patientenanzahl & Anzahl & $\%$ \\
\hline \multirow[t]{2}{*}{ Geschlecht } & männlich & 16 & 64 \\
\hline & weiblich & 9 & 36 \\
\hline \multirow[t]{3}{*}{ Alter } & Mittelwert & 49 & Jahre \\
\hline & Minimum & 14 & Jahre \\
\hline & Maximum & 72 & Jahre \\
\hline Lokalisation des & Kopf/Hals & 6 & 24 \\
\hline \multirow[t]{3}{*}{ Primärtumors } & Extremitäten & 12 & 48 \\
\hline & Stamm & 5 & 20 \\
\hline & okkult & 2 & 8 \\
\hline \multirow{8}{*}{$\begin{array}{l}\text { AJCC Stadium- } \\
\text { Anzahl }\end{array}$} & IB & 4 & 16 \\
\hline & $\| \mathrm{A}$ & 3 & 12 \\
\hline & IIB & 6 & 24 \\
\hline & IIIA & 2 & 8 \\
\hline & IIIB & 4 & 16 \\
\hline & IIIC & 2 & 8 \\
\hline & IV & 2 & 8 \\
\hline & nicht bestimmbar* & 2 & 8 \\
\hline \multirow{2}{*}{$\begin{array}{l}\text { Sentinel lymph node } \\
\text { Biopsie }\end{array}$} & erfolgt & 8 & 32 \\
\hline & nicht erfolgt & 17 & 68 \\
\hline \multirow{2}{*}{ Lymphknotenbefall } & ja & 11 & 44 \\
\hline & nein & 14 & 56 \\
\hline
\end{tabular}

* Lymphknotenbefall bei okkultem Primarius.

rung und schriftlicher Einverständniserklärung erhielten alle Patienten $2 \mu \mathrm{g} / \mathrm{kg}$ Körpergewicht PEG-IFN- $\alpha-2 \mathrm{~b}$ subkutan $1 \times /$ Woche. Die Therapiedauer wurde auf 18 Monate festgelegt.

Primärer Endpunkt der Analyse war die Erfassung der Inzidenz von Auto-AK und Autoimmunerkrankungen unter PEG-IFN$\alpha$-2b-Therapie. Patienten mit Nachweis von AK vor Beginn der Therapie wurden für die Datenanalyse nicht berücksichtigt. Die sequenziellen serologischen Untersuchungen schlossen antinukleäre Antikörper (ANA), TSH, fT3, fT4, anti-Thyreoglobulin-AK (anti-TG-AK), anti-TSH-Rezeptor-AK (anti-TSH-R-AK) und antiThyreoidea-Peroxidase-AK (TPO-AK) ein und erfolgten vor sowie alle drei Monate nach Beginn der Behandlung. Der ANA-Screen erfolgte mittels indirekter Immunfluoreszenz auf Hep-2-Zellen mit weiterer Differenzierung ab einem Titer von 1:80 mittels ELISA in dsDNS-AK; RNP-AK, Sm-AK, Ro-AK, La-AK, Scl-70-AK, CENP-B-AK und Jo-1-AK. Die Bestimmung der Schilddrüsen-AK erfolgte ebenfalls mittels ELISA.

Klinische Untersuchungen erfolgten vor Beginn sowie alle drei Monate im Verlauf der Therapie. Zur Erfassung eines Tumorprogresses wurden Staginguntersuchungen mittels CT-Thorax, CTAbdomen und MRT-Kopf in halbjährlichen Abständen durchgeführt, die sonografische Kontrolle der drainierenden Lymphknoten erfolgte vierteljährlich. Die Endpunktanalyse zum progressionsfreien und Gesamtüberleben erfolgte 36 Monate nach Beginn der adjuvanten Immuntherapie.

\section{Ergebnisse \\ $\nabla$}

Bei 33 Patienten wurden vor Beginn der PEG-IFN- $\alpha$-2b-Therapie Serumproben auf das Vorhandensein von Auto-AK untersucht, acht Patienten wurden von der weiteren Analyse ausgeschlossen, da bereits vor Beginn der Therapie Auto-AK detektiert werden konnten. Das mittlere Alter der 25 auswertbaren Patienten lag bei 49 Jahren (Minimum 14 Jahre, Maximum 72 Jahre). Bei Behandlungsbeginn waren vier Patienten (16\%) im AJCC Stadium IB, neun (36\%) im Stadium II, acht Patienten (32\%) im Stadium III und zwei Patienten im Stadium IV (8\%), Stadium III und 
Tab. 2 Zeit bis zur Progression und Überlebensstatus in Assoziation zur Autoimmuninduktion unter adjuvanter Therapie mit PEG-IFN- $\alpha$-2b

\begin{tabular}{|c|c|c|c|c|c|c|c|c|}
\hline Pat.Nr. & Stadium & ANA & $\begin{array}{l}\text { ANA-Diffe- } \\
\text { renzierung }\end{array}$ & $\begin{array}{l}\text { Schilddrüsen- } \\
\text { AK }\end{array}$ & $\begin{array}{l}\text { Autoimmun- } \\
\text { thyreoiditis }\end{array}$ & $\begin{array}{l}\text { Zeit bis zur } \\
\text { Progression }\end{array}$ & $\begin{array}{l}\text { Überlebens- } \\
\text { status }\end{array}$ & $\begin{array}{l}\text { Überlebens- } \\
\text { zeitraum }\end{array}$ \\
\hline 1 & IV & + & SSA-AK & + & - & 2 Monate & lebt & 36 Monate \\
\hline 2 & Ib & + & - & + & + & nicht erreicht & lebt & 36 Monate \\
\hline 3 & IV & + & - & + & + & 4 Monate & lebt & 36 Monate \\
\hline 4 & IIb & - & - & - & - & nicht erreicht & lebt & 36 Monate \\
\hline 5 & IIIC & + & - & - & - & 13 Monate & verstorben & 28 Monate \\
\hline 6 & $\mathrm{lb}$ & + & - & - & - & nicht erreicht & lebt & 36 Monate \\
\hline 7 & IIIb & + & SSA-AK & - & - & nicht erreicht & lebt & 36 Monate \\
\hline 8 & $\mathrm{Ib}$ & - & - & + & + & nicht erreicht & lebt & 36 Monate \\
\hline 9 & Ilb & + & - & - & - & 14 Monate & verstorben & 24 Monate \\
\hline 10 & IIA & - & - & - & - & nicht erreicht & lebt & 36 Monate \\
\hline 11 & okkult & - & - & - & - & 12 Monate & lebt & 36 Monate \\
\hline 12 & IIIC & - & - & - & - & 6 Monate & verstorben & 18 Monate \\
\hline 13 & Ila & + & - & - & - & nicht erreicht & lebt & 36 Monate \\
\hline 14 & IIb & + & - & - & - & nicht erreicht & lebt & 36 Monate \\
\hline 15 & IIb & + & - & - & - & 11 Monate & verstorben & 25 Monate \\
\hline 16 & Ila & + & $\begin{array}{l}\text { DsDNS-AK, } \\
\text { SSA-AK, } \\
\text { SCl-70-AK }\end{array}$ & + & - & nicht erreicht & lebt & 36 Monate \\
\hline 17 & IIIa & - & - & - & - & 6 Monate & verstorben & 15 Monate \\
\hline 18 & IIb & - & - & - & - & nicht erreicht & lebt & 36 Monate \\
\hline 19 & okkult & - & - & - & - & 29 Monate & verstorben & 35 Monate \\
\hline 20 & IIb & + & - & - & - & nicht erreicht & lebt & 36 Monate \\
\hline 21 & IIIb & + & - & - & - & 12 Monate & verstorben & 16 Monate \\
\hline 22 & $\mathrm{IIIb}$ & + & - & - & - & 15 Monate & verstorben & 21 Monate \\
\hline 23 & IIIb & + & - & - & - & 28 Monate & lebt & 36 Monate \\
\hline 24 & $\mathrm{lb}$ & - & - & - & - & 24 Monate & lebt & 36 Monate \\
\hline 25 & IIIa & - & - & - & - & nicht erreicht & lebt & 36 Monate \\
\hline
\end{tabular}

IV jeweils nach R0-Resektion ( $\bullet$ Tab. 1). Zwei Patienten (8\%) mit operiertem Lymphknotenbefall bei okkultem Primarius wurden als nicht klassifizierbar eingestuft. Im Beobachtungszeitraum von 36 Monaten entwickelten 13 der 25 Patienten einen Progress (52\%), wovon acht Patienten innerhalb des Beobachtungszeitraumes verstarben (32\%) ( Tab. 2).

Unter Therapie entwickelten 16 von 25 Patienten (64\%) Auto-AK mit unterschiedlicher Titerhöhe und Profil ( Tab.3). ANA konnten bei 15 Patienten nachgewiesen werden. Eine Differenzierung der ANA erbrachte bei drei Patienten den Nachweis von SSA-AK, bei einem Patienten wurden zusätzlich dsDNS-AK und Scl-70-AK detektiert, jedoch ohne klinische Hinweise auf das Vorliegen einer Kollagenose. Schilddrüsen-AK konnten bei fünf Patienten nachgewiesen werden. Vier dieser Patienten entwickelten anti-TG-AK, zwei zusätzlich TPO-AK; beim fünften Patienten wurden ausschließlich TSH-R-AK detektiert. Drei dieser fünf Patienten entwickelten unter der Therapie mit PEG-IFN$\alpha-2 b$ eine klinisch manifeste Autoimmunthyreoiditis.

Bei 56\% ( $n=5 / 9)$ AK-negativen Patienten trat im Median 15,4 Monate nach Beginn der IFN-Therapie eine Progression auf. Nur vier Patienten (44\%) entwickelten im Beobachtungszeitraum von 36 Monaten keine Progression. In der AK-negativen Gruppe waren nach 36 Monaten noch 67\% der Patienten am Leben. Die mittlere Überlebensdauer bei einer Endpunktsetzung bei 36 Monaten betrug 31,6 Monate.

Bei Patienten mit nachgewiesener AK-Induktion unter Therapie lagen Progressionsrate wie auch die Rate der persistierenden Remissionen bei 50\% (jeweils acht von 16 Patienten). Die durchschnittliche Zeit bis zur Progression betrug in diesem Kollektiv 12,4 Monate. Von diesen Patienten waren nach 36 Monaten
Tab. 3 Unter adjuvanter PEG-IFN- $\alpha$-2b induziertes Antikörperprofil

\begin{tabular}{lll}
$\begin{array}{l}\text { Antikörper- } \\
\text { typ }\end{array}$ & $\begin{array}{l}\text { Patienten- } \\
\text { anzahl }\end{array}$ & $\begin{array}{l}\text { Antikörperhäufigkeit bezogen } \\
\text { auf die Patientengesamtzahl in } \\
\text { Prozent (\%) }\end{array}$ \\
\hline ANA & 15 & 60 \\
\hline SSA-AK & 3 & 12 \\
\hline SCl-70-AK & 1 & 4 \\
\hline DSDNS-AK & 1 & 4 \\
\hline TG-AK & 4 & 16 \\
\hline TSH-R-AK & 1 & 4 \\
\hline TPO-AK & 2 & 8 \\
\hline
\end{tabular}

noch 69\% der Patienten am Leben. Die mittlere Überlebensdauer betrug 31,9 Monate.

Der Überlebensstatus des Gesamtkollektivs der Patienten mit AK-Induktion unterschied sich im Vergleich zur AK-negativen Gruppe nur unwesentlich ( Tab. 2). Herauszuheben ist jedoch, dass keiner der sechs Patienten, die unter IFN-Therapie eine klinisch manifeste Autoimmunthyreoiditis entwickelten und/oder bei denen spezifische ANA in der ANA-Differenzierung nachgewiesen werden konnten, im Beobachtungszeitraum von 36 Monaten nach Beginn der Therapie verstorben ist ( $\mathbf{A b b}$. 1). Bemerkenswert ist, dass zwei dieser sechs Patienten die adjuvante Immuntherapie im Stadium IV der Erkrankung erhielten und unter IFN-Therapie resezierbare Tumorrezidive entwickelten. Verglichen hierzu lag die mittlere Überlebenszeit der AK-positiven Patienten ohne spezifische AK oder Autoimmunerkrankung bei nur 26,73 Monaten (> 36 Monate versus 26,73 Monate), bei einer Sterberate von 50\% (5/10) im Beobachtungszeitraum von 36 Monaten $(\bullet$ Abb. 1) 


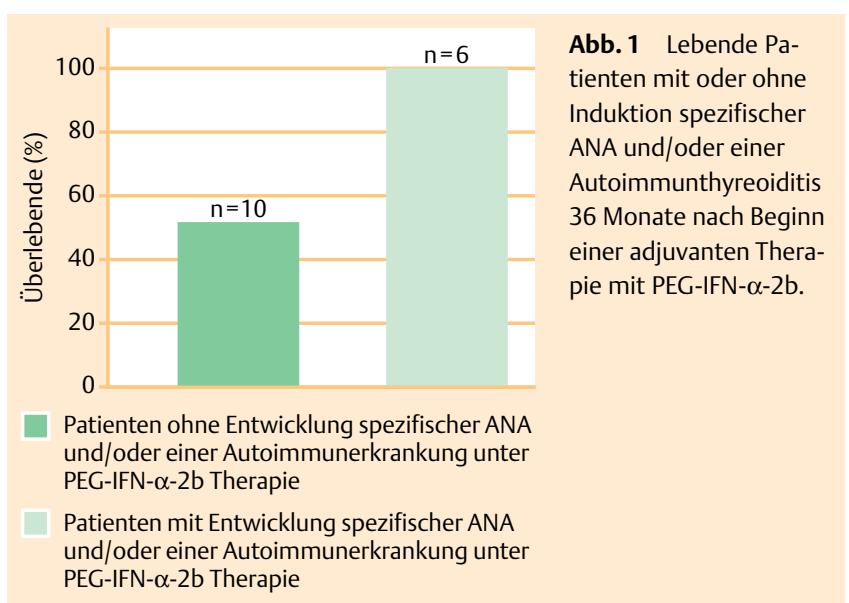

\section{Diskussion}

$\nabla$

Das Auftreten von Autoimmunphänomenen wird bei Patienten, die an einer Tumorerkrankung leiden, mit einer besseren Prognose assoziiert, Untersuchungen hierzu liegen insbesondere zum malignen Melanom und dem Nierenzellkarzinom vor $[4,9,10]$. Beim malignen Melanom wird seit Langem kasuistisch über ein verlängertes Überleben beim Auftreten vitiligoähnlicher Hypopigmentierungen in Primärtumoren oder in disseminierter Form als paraneoplastisches Syndrom im Stadium der Metastasierung berichtet [17,18]. Prospektive randomisierte Studien, die untersuchen, ob Melanompatienten mit endogener Autoimmunstimulation einen günstigeren Krankheitsverlauf zeigen, stehen bisher aus.

Eine Assoziation zwischen dem Auftreten einer Autoimmunität und einem antitumorösen Effekt konnte in der Behandlung des malignen Melanoms neben der adjuvanten IFN-Therapie auch für andere Formen der Immuntherapie gezeigt werden, insbesondere für eine Therapie mit Interleukin-2 und CTLA-4 AK $[19,20]$. Daten zur Häufigkeit von Autoimmunphänomenen unter adjuvanter Therapie mit PEG-IFN- $\alpha$ und deren Korrelation zum progressionsfreien und Gesamtüberleben liegen zum malignen Melanom bisher nicht vor.

In unserer retrospektiven Analyse von 25 Patienten, die aufgrund eines malignen Melanoms adjuvant mit PEG-IFN- $\alpha-2 b$ behandelt wurden, fanden wir, dass $64 \%$ der Patienten unter Therapie Auto-AK entwickelten. Dies liegt deutlich über den publizierten Daten zu nicht pegyliertem IFN- $\alpha$. Gogas et al. konnten unter adjuvanter Hochdosis-IFN-Therapie beim malignen Melanom im AJCC Stadium II und III bei 26\% der Patienten AutoAK oder eine klinisch manifeste Autoimmunerkrankung diagnostizieren [9]. An einer manifesten Autoimmunerkrankung der Schilddrüse erkrankten in unserem Kollektiv 16\% der Patienten, im Vergleich zu den von Gogas benannten 7\% [9]. Weitere Autoimmunerkrankungen wie Vitiligo, Rheumatoide Arthritis oder Kollagenosen traten bei unseren Patienten unter adjuvanter Immuntherapie mit PEG-IFN- $\alpha$-2b nicht auf.

Bei einer Nachbeobachtungszeit von 36 Monaten bestand kein Unterschied zwischen AK-positivem und AK-negativem Kollektiv bezüglich der Zeit bis zur Progression, der Progressionsrate und der Überlebensrate. Zu betonen ist, dass alle Patienten mit einer klinisch manifesten Autoimmunthyreoiditis und/oder dem Nachweis spezifischer ANA nach 36 Monaten noch lebten. Besonders herauszuheben ist, dass zwei dieser Patienten im R0-resezierten Stadium IV der Erkrankung behandelt wurden und trotz mehrfacher Tumorrezidive am Ende des 36-monatigen Beobachtungszeitraumes am Leben waren.

Im Gegensatz zu den Untersuchungen bei Gogas et al. zum nicht pegylierten IFN- $\alpha$ [9] konnte in unserem Patientenkollektiv unter PEG-IFN- $\alpha-2 b$ zwar ein höherer Anteil von Auto-AK detektiert werden, relevant zur Induktion einer kontrollierenden Immunantwort gegen Tumorgewebe schien jedoch die Stärke und Spezifität der Autoimmunreaktion zu sein, die sich in einer klinisch manifesten Autoimmunthyreoiditis und/oder dem Nachweis hochtitriger spezifischer Auto-AK widerspiegelte.

Eine positive Korrelation von Autoimmunität und Tumortherapie wird hypothetisch mit der Immunstimulation durch Autoantigene als Ursache der antitumoralen Antwort in Zusammenhang gebracht. Ähnlich wird auch die Kreuzreaktivität von AK gegen Autoantigen und Melanomantigen diskutiert. Möglicherweise unterliegt die Immunantwort gegenüber dem Gewebe, gegen das die Autoimmunantwort gerichtet ist, einer ähnlichen Regulation wie der Immunantwort auf das Tumorgewebe. Die Hypothese einer spezifischen Kreuzreaktivität könnte erklären, warum bei unserer Untersuchung die Induktion von ANA ohne spezifische Differenzierung wenig relevant für eine dauerhafte Tumorkontrolle zu sein schien.

Da die Entwicklung einer Autoimmunreaktion mittels IFN zuweilen erst nach mehrmonatiger Therapie auftritt, kann die Induktion spezifischer Auto-AK oder Autoimmunerkrankungen unter IFN-Therapie nicht als Surrogatmarker zur Selektion von Patienten dienen, die von einer IFN-Therapie profitieren würden. Patienten mit dokumentierter vorbestehender Neigung zur Autoimmunität könnten hingegen eine Patientengruppe sein, bei denen eine Immuntherapie indiziert sein könnte, vorausgesetzt, dass die Autoimmunität beherrschbar ist.

Wünschenswert wäre die Selektion von Patienten mittels Biomarkern vor Beginn der IFN-Therapie, bei denen unter Behandlung eine Autoimmunität induziert werden kann. Polymorphismen im CTLA-4 Gen oder Mutationen im FOXp3 Transkriptionsfaktor, als kritische Regulationswege der T-Zellen, aber auch Assoziationen zwischen HLA-Genotypen und bestimmten Autoimmunerkrankungen könnten in der Identifizierung solcher Biomarker helfen. So fanden Franzke et al. beim metastasierten Nierenzellkarzinom eine Assoziation zwischen HLA-Cw7 positiven Patienten, einer Induktion von Schilddrüsen-AK und einer Verlängerung des Gesamtüberlebens nach Therapie mit IFN- $\alpha$ und Interleukin-2 [10]. Ebenfalls bei Patienten mit Nierenzellkarzinom kann die Expression von Carboanhydrase IX das Anprechen auf eine Immuntherapie mit Interleukin-2 vorhersagen [21].

Die prospektive Identifikation von Patienten mit immunsensitiven Tumoren, mit induzierbarer protektiver antitumoraler Immunantwort mittels spezifischen Biomarkern, würde aber nicht nur Patienten für eine Immuntherapie selektieren, sondern ebenfalls Patienten, die von einer Immuntherapie nicht profitieren würden, eine potenziell nebenwirkungsreiche Behandlung ersparen. Die Erkenntnisse zur Autoimmunität unter adjuvanter IFN-Therapie und die Assoziation zum verbesserten Tumoransprechen sind erste Schritte in der Entwicklung patientenspezifischer Behandlungsstrategien beim malignen Melanom. 
Abstract

Induction of Autoimmunity by Pegylated Interferonalpha-2b in Correlation to Survival Time in Medium and High Risk Melanoma Patients

$\nabla$

Background: The appearance of autoimmunity in tumor patients seems to be associated with a better prognosis. The aim of this retrospective study was to detect autoimmunity under adjuvant treatment with pegylated interferon-alpha- $2 \mathrm{~b}$ and to determine a correlation between autoimmune induction and survival.

Patients and Methods: To detect autoimmunity clinical and serological parameters were examined in 25 patients with R0-resected histological proven stage Ib-IV melanoma before and every three months during an 18-month adjuvant treatment with $2 \mu \mathrm{g} / \mathrm{kg}$ pegylated interferon-alpha-2b given once a week. Results: Under treatment 16 of 25 patients (64\%) developed autoantibodies. There was no significant difference regarding survival data in patients with or without induction of autoantibodies (31,6 versus 31,9 months). However, it is remarkable that all 6 patients developing a clinically significant autoimmune thyroiditis and/or specific antinuclear antibodies were still alive 36 months after treatment beginning, with two of these patients being in complete remission from stage IV melanoma, compared to a mean survival time of 26,73 months for the remaining patient group with physiological irrelevant positive antibodies $(n=10 / 16)$.

Conclusions: In our cohort, the number of patients who developed autoimmunity under pegylated interferon-alpha-2b is significantly higher as published data of non-pegylated interferon-alpha. Nevertheless, the significance of specific autoimmune induction as a prognostic indicator for a disease controlling antitumor immune response still needs to be confirmed.

\section{Literatur}

1 Scheibenbogen C, Hunstein W, Keilholz U. Vitiligo-like lesions following immunotherapy with IFN alpha and IL-2 in melanoma patients. Eur J Cancer 1994; 30: 1209-1211

2 Guillot B, Blazquez L, Bessis D, Dereure O, Guilhou JJ. A prospective study of cutaneous adverse events induced by low-dose alpha-interferon treatment for malignant melanoma. Dermatology 2004; 208: 49-54

3 Kabbaj N, Guedira MM, El Atmani H, El Alaoui M, Mohammadi M, Benabed $K$, Lachkar H, Benaissa A. Thyroid disorders during interferon alpha therapy in 625 patients with chronic hepatitis C: a prospective cohort study. Ann Endocrinol 2006; 67: 343 - 347

4 Satzger I, Meier A, Schenck F, Kapp A, Hauschild A, Gutzmer R. Autoimmunity as a prognostic factor in melanoma patients treated with adjuvant low-dose interferon alpha. Int J Cancer 2007; 121: 2562 - 2566

5 Valenti G, Mantelli A, Persico M, Tuccillo C, Del Vechio Blanco G, Morisco F, Improta R, Migliaresi S, Gualdieri L, Caporaso N. Serologic and clinical markers of autoimmune disease in HCV-infected subjects with different disease conditions. Clin Exp Rheumatol 1999; 17: 75 - 79

6 Clifford BD, Donahue D, Smith L, Cable E, Luttig B, Manns M, Bonkovsky $H L$. High prevalence of serologic markers of autoimmunity in patients with chronic hepatitis C. Hepatology 1995; 21: 613-619
7 Wandl UB, Nagel-Hiemke M, May D, Kreutzfelder E, Kloke O, Kranzhoff $M$, Seeber S, Niederle N. Lupus-like autoimmune disease induced by interferon therapy for myeloproliferative disorders. Clin Immunol Immunopathol 1992; 65: 70-74

8 Toubi E, Shoenfeld Y. Protective autoimmunity in cancer. Oncol Rep 2007; 17: $245-51$

9 Gogas H, Ioannovich J, Dafni U, Stavropoulou-Giokas C, Frangia K, Tsoutsos D, Panagiotou P, Polyzos A, Papadopoulos O, Stratigos A, Markopoulos C, Bafaloukos D, Pectasides D, Fountzilas G, Kirkwood JM. Prognostic significance of autoimmunity during treatment of melanoma with interferon. N Engl J Med 2006; 354: 709-718

10 Franzke A, Peest D, Probst-Kepper M, Buer J, Kirchner GI, Brabant G, Kirchner H, Ganser A, Atzpodien J. Autoimmunity resulting from cytokine treatment predicts long-term survival in patients with metastatic renal cell cancer. J Clin Oncol 1999; 17: 529-533

11 Attia P, Phan GQ, Maker AV, Robinson MR, Qezado MM, Yang JC, Sherry RM, Topalian SL, Kammula US, Royal RE, Restifo NP, Haworth LR, Levy C, Mavroukakis SA, Nichol G, Yellin MJ, Rosenberg SA. Autoimmunity correlates with tumor regression in patients with metastatic melanoma treated with anti-cytotoxic T-lymphocyte antigen-4. J Clin Oncol 2005; 23: 6043-6053

12 Glue P, Fang JW, Rouzier-Panis R, Raffanel C, Sabo R, Gupta SK, Salfi M, Jacobs $S$. Pegylated interferon-alpha2b: pharmacokinetics, pharmacodynamics, safety and preliminary efficacy data. Hepatitis C Intervention Therapy Group. Clin Pharmacol Ther 2000; 68: 556 - 567

13 Lindsay KL, Trepo C, Heintges T, Shiffman ML, Gordon SC, Hoefs JC, Schiff ER, Goodman ZD, Laughlin M, Yao R, Albrecht JK, Hepatitis Interventional Therapy Group. A randomized, double-blind trial comparing pegylated interferon alfa- $2 \mathrm{~b}$ to interferon alfa-2b as initial treatment for chronic hepatitis C. Hepatology 2001; 34: 395-403

14 Manns MP, McHutchison JG, Gordon SC, Rustgi VK, Shiffman M, Reindollar R, Goodman ZD, Koury K, Ling M, Albrecht JK. Peginterferon alfa-2b plus ribavirin compared with interferon alfa-2b plus ribavirin for initial treatment of chronic hepatitis C: a randomised trial. Lancet 2001; 358: $958-965$

15 Talpaz M, O'Brien S, Rose E, Gupta S, Shan J, Cortes J, Giles FJ, Faderl S, Kantarjian HM. Phase 1 study of polyethylene glycol formulation of interferon alpha-2b (Schering 54031) in Philadelphia chromosomepositive chronic myelogenous leukemia. Blood 2001; 98: 1708-1713

16 Bukowski R, Ernstoff MS, Gore ME, Neumunaitis IJ, Amato R, Gupta SK, Tendler $C L$. Pegylated Interferon alfa- $2 \mathrm{~b}$ treatment for patients with solid tumors a phase I/II study. J Clin Oncol 2002; 20: 3841 - 3849

17 Bystryn JC, Riegel D, Friedman RJ, Kopf A. Prognostic significance of hypopigmentation in malignant melanoma. Arch Dermatol 1987; 123: $1053-1055$

18 Nordlung JJ, Kirkwood JM, Forget BM, Milton G, Albert DM, Lerner AB. Vitiligo in patients with metastatic melanoma: a good prognostic sign. J Am Acad Dermatol 1983; 9: 689-696

19 Phan GQ Attia P, Steinberg SM, White DE, Rosenberg SA. Factors associated with response to high-dose interleukin-2 in patients with metastatic melanoma. J Clin Oncol 2001; 19: 3477-3482

20 Phan GQ Yang JC, Sherry RM, Hwu P, Topalian SL, Schwartzentruber DJ, Restifo NP, Haworth LR, Seipp CA, Freezer LJ, Morton KE, Mavroukakis SA, Duray PH, Steinberg SM, Allison JP, Davis TA, Rosenberg SA. Cancer regression and autoimmunity induced by cytotoxic $T$ lymphocyteassociated antigen 4 blockade in patients with metastatic melanoma. Proc Natl Acad Sci USA 2003; 100: 8372 - 8377

21 Atkins M, Regan M, McDermott D, Mier J, Stanbridge E, Youmans A, Febbo P, Upton M, Lechpammer M, Signoretti S. Carbonic anhydrase IX expression predicts outcome of interleukin-2 therapy for renal cancer. Clin Cancer Res 2005; 11: 3714 - 3721 